\title{
Sintilimab: A Promising Anti-Tumor PD-1 Antibody
}

\author{
Lin Zhang ${ }^{1 \dagger}$, Wuqian $\mathrm{Mai}^{2,3+}$, Wenyang Jiang ${ }^{1 *}$ and Qing Geng ${ }^{1 *}$ \\ ${ }^{1}$ Department of Thoracic Surgery, Renmin Hospital of Wuhan University, Wuhan, China, ${ }^{2}$ Department of Cardiology, Union \\ Hospital, Tongji Medical College, Huazhong University of Science and Technology, Wuhan, China, ${ }^{3}$ Key Lab of Molecular \\ Biological Targeted Therapies of the Ministry of Education, Union Hospital, Tongji Medical College, Huazhong University of \\ Science and Technology, Wuhan, China
}

\section{OPEN ACCESS}

Edited by:

Jose A. Garcia-Sanz, Consejo Superior de Investigaciones Cientificas (CSIC), Spain

Reviewed by:

Shinichi Makita,

National Cancer Center Hospital, Japan

Graham Robert Leggatt, The University of Queensland, Australia

Jean-Marie Michot, Institut Gustave Roussy, France

*Correspondence: Qing Geng gengqingwhu@whu.edu.cn Wenyang Jiang whujwy0228@163.com

${ }^{+}$These authors have contributed equally to this work

Specialty section:

This article was submitted to Cancer Immunity and Immunotherapy,

a section of the journal

Frontiers in Oncology

Received: 17 August 2020

Accepted: 27 October 2020

Published: 26 November 2020

Citation:

Zhang L, Mai W, Jiang $W$ and Geng $Q$ (2020) Sintilimab: A Promising AntiTumor PD-1 Antibody.

Front. Oncol. 10:594558. doi: 10.3389/fonc.2020.594558
Sintilimab $\left(\right.$ Tyry $\left.{ }^{\circledR}\right)$ is a monoclonal antibody against programmed cell death protein 1 (PD-1). It could block the interaction between PD-1 and its ligands and help the anti-tumor effect of Tcells to recover. Sintilimab is developed by Innovent Biologics and Eli Lilly and Company and has been approved to treat relapsed or refractory classical Hodgkin lymphoma in patients who have undergone two or more lines of systemic chemotherapy by the National Medical Products Administration of China. Recently, sintilimab has been reported in plenty of literature and shows satisfying anti-tumor effect. Meanwhile, there are some reports showing its side effects. Overall, sintilimab has similar anti-tumor effects and a better safety profile compared to nivolumab and pembrolizumab in Hodgkin lymphoma, natural killer/T cell lymphoma and advanced non-small cell lung cancer. In this review, we aim to briefly describe the mechanisms, pharmacological characteristics, anti-tumor effects, predictive parameters of efficacy and side effects of sintilimab, providing valuable information of sintilimab for decisionmaking in the treatment of tumors in the future.

Keywords: sintilimab, programmed cell death protein 1//programmed cell-death protein 1 ligand 1, immunotherapy, non-small cell lung cancer, solid tumors, Hodgkin lymphoma

\section{INTRODUCTION}

Immune checkpoints (ICs) are important immune regulators in maintaining immune homeostasis and preventing autoimmune diseases. There are co-stimulatory ICs, like CD28 and TNFRSF9, and inhibitory ICs, like CTLA-4 (1). Under normal conditions, ICs allow the immune system to respond to infections, malignancies and protect tissues. However, the expression of some immune checkpoint proteins by tumor cells disturbs the anti-tumor immunity of the body, facilitating the growth and expansion of malignant cells (2). Thus, blockade of the immune checkpoint pathway using immune checkpoint inhibitors (ICIs) is a weapon in cancer therapy. Among all ICIs, the most studied ones are anti-programmed cell death protein 1(PD-1)/programmed cell-death protein 1 ligand 1(PD-L1) antibodies (3).

Sintilimab is a fully human IgG4 monoclonal antibody that is registered as Tyvyt. It was developed by Innovent Biologics and Eli Lilly and Company (4). Sintilimab is able to bind to PD-1, block the interaction of PD-1 with its ligands, and help to recover the anti-tumor response of Tcells. By 2020, sintilimab has been approved by the National Medical Products Administration (NMPA) of China to treat relapsed or refractory classical Hodgkin lymphoma $(\mathrm{r} / \mathrm{r} \mathrm{cHL})$ (5). 
Compared with nivolumab and pembrolizumab, two wellstudied PD-1 inhibitors approved by China NMPA and the US Food and Drug Administration (FDA), sintilimab has a similar anti-tumor effect, a better safety profile, and obvious economic advantages. Here, we summarized the mechanisms, pharmacological characteristics, anti-tumor effects, predictive parameters of efficacy and adverse effects (AEs) of this drug to provide a potential valuable choice for decision-making in the treatment of tumors in the future.

\section{PD-1/PD-L1 PATHWAY}

PD-1 was first characterized in 1992 by Ishida et al. of Kyoto University in Japan (6). As one of the immune checkpoint membrane proteins, PD-1 is synthesized in the endoplasmic reticulum and then delivered to the cell membrane surface to play inhibitory roles (3). Structurally, PD-1 is a type I transmembrane protein that belongs to the CD28 immunoglobulin (Ig) subfamily and is an important immunosuppressive protein (7). PD-1 consists of an Ig-V like extracellular domain, a transmembrane domain, and a cytoplasmic domain; the cytoplasmic domain is comprised of two tyrosine signaling motifs, which are known as immunoreceptor tyrosine inhibitory motif (ITIM) and immunoreceptor tyrosine-based switch motif (ITSM) $(6,8)$. Previous research has indicated that the tyrosine within the ITSM motif is indispensable for the function of PD-1 in T cells and $\mathrm{B}$ cells $(9,10)$. PD-1 could recruit protein tyrosine phosphatase SHP2/SHP1 after binding with its ligands; the process was mediated via phosphorylated ITSM/ITIM, which could in turn inhibit both T cell receptor (TCR) and CD28 (3, 11). The downstream phosphatidylinositol 3 kinase (PI3K)/ protein kinase $\mathrm{B}(\mathrm{AKT})$ pathway of CD28 and signaling molecules like zeta-chain associated protein 70 of TCR were then inhibited (3). The ligands of PD-1 included PD-L1 and PDL2 $(12,13)$. The expression of PD-L2 on tumor cells is rare, and the role of $\mathrm{PD}-1 / \mathrm{PD}-\mathrm{L} 1$ pathway in the regulation of anti-tumor immunity is more studied (14).

The PD-1/PD-L1 pathway is involved in the regulation of tumor progression in many aspects. The expression of PD-L1 was mainly induced by interferon- $\gamma($ IFN- $\gamma)$, and the primary mechanism of PD-1/PD-L1 pathway blockade is generally thought to be the priming of tumor-specific cytotoxic $\mathrm{T}$ cells in the tumor microenvironment $(11,15)$. The binding of PD-L1 and PD-1 could lead to T cell apoptosis, anergy, exhaustion, and interleukin-10 expressions $(15,16)$. PD-L1 may also function as a molecular "shield" to protect malignant cells from cytotoxic responses mediated by $\mathrm{CD}^{+} \mathrm{T}$ cells $(15,17)$. Activated $\mathrm{T}$ cells and antigen-presenting cells could express CD80, a receptor of PD-L1, delivering inhibitory signals when binding to it (16). In addition, PD-L1 might act as a receptor to transmit signals from $\mathrm{T}$ cells to tumor cells, resulting in their resistance to lysis (17). Tumor-infiltrating lymphocyte (TIL) is a major effector cell in the microenvironment of tumor tissues, and it highly expresses PD-1 molecules on the surface (15). TIL inactivation is a consequence of PD-1/PD-L1 activation since silencing PD-1 and PD-L1 could effectively increase TIL cytotoxicity to cancer cells (18). Finally, the overexpression of PD-1 in tumor cells could promote the growth of tumors (19).

\section{PHARMACOLOGICAL CHARACTERISTICS OF SINTILIMAB}

Sintilimab has an effective anti-tumor activity. In vitro, the binding affinity of sintilimab was high with a low dissociation constant (KD) $(20,21)$. Correspondingly, sintilimab has shown a higher level of PD-1 occupancy than nivolumab and pembrolizumab in peripheral blood mononuclear cells (PBMCs) and mice reconstituted with PBMCs (20). In patients, a sustained $\mathrm{PD}-1$ receptor occupancy of more than $95 \%$ for up to 4 weeks was observed after a single sintilimab infusion intravenously (20). Sintilimab could effectively activate $\mathrm{T}$ cells, as evidenced by the enhanced interleukin- 2 and IFN- $\gamma$ secretion among $\mathrm{T}$ cells co-incubated with it $(20,21)$. PD-1 is inducibly expressed on activated $\mathrm{T}$ cells but is not expressed on resting $\mathrm{T}$ cells (22). In a human tumor xenograft mice model reconstituted with human immune cells or a PD-1 knockin tumor mouse model, sintilimab could significantly inhibit tumor growth and the effect was more prominent than equal doses of nivolumab or pembrolizumab $(20,21)$.

Sintilimab could be metabolized effectively and has an acceptable safety profile in the body. The serum half-life $\left(t_{1 / 2}\right)$ of sintilimab is $35.6 \mathrm{~h}$ compared to those of $43.5 \mathrm{~h}$ and $42.5 \mathrm{~h}$ for nivolumab and pembrolizumab, respectively (20). The concentration for $50 \%$ of maximal effect (EC50) is $2.2 \mathrm{nM}$ (21). Like other therapeutic antibodies, it has an IgG4 backbone, which is known to have a very low effector function. In fact, sintilimab has no or weak affinity to Fc receptors for IgG $(\mathrm{Fc} \gamma \mathrm{R})$, implicating that sintilimab does not induce antibody or complement-dependent cytotoxic responses (21). Also, sintilimab has a weak immunogenicity as reflected by low detection rates of anti-drug antibodies $(0.52 \%, 2 / 381)$ and neutralizing antibodies $(0.26 \%, 1 / 381)$ in patients. The toxicity of sintilimab was tolerable in a 26-week toxicity study in which sintilimab was administrated biweekly up to $200 \mathrm{mg} / \mathrm{kg}$ and caused no death among cynomolgus monkeys (21). However, a potential risk of IgG4 antibodies is Fab-arm exchange (FAE), which means the swap of a heavy chain and the attachment of light chain with a heavy-light chain pair from another molecule in IgG4 antibody; FAE could lead to molecular instability (23). Luckily, FAE could be eliminated by antibody engineering with a single point mutation (24).

\section{EFFICACY OF SINTILIMAB IN TUMORS}

In a first-in-human phase I trial (NCT02937116), sintilimab has shown preliminary efficacy and tolerance among 12 patients with solid tumors including hepatocellular carcinoma and neuroendocrine tumor (25). In that study, sintilimab was given at escalating doses: 1, 3, 200 (1:1 randomization) and $10 \mathrm{mg} / \mathrm{kg}$ 
Finally, a dose of $200 \mathrm{mg}$ per 3 weeks (q3w) was recommended for further studies (25).

\section{Efficacy of Sintilimab in Lymphoma}

Sintilimab monotherapy has shown anti-tumor efficacy in lymphoma. The first indication of sintilimab is relapsed or refractory classical Hodgkin lymphoma after a second-line chemotherapy, which was a conditioned approval based on a multicenter, single-arm, phase II trial (ORIENT-1) $(26,27)$. In ORIENT-1, 96 patients with $\mathrm{r} / \mathrm{r} \mathrm{cHL}$ after $\geq 2$ lines of therapy from 18 hospitals in China were incorporated with 92 included in the full analysis (26). Patients were given intravenous sintilimab $200 \mathrm{mg}$ q3w until progression, death, unacceptable toxicity, or withdrawal of consent; among them, 74 patients $(80.4 \% ; 74 / 92)$ had an objective response (OR), defined as complete or partial remission on CT or MRI, according to an independent radiological review committee $(26,28)$. In comparison, 66.3\% (53/80) and 69.0\% (145/210) r/r cHL patients achieved OR after treatment with nivolumab $(3 \mathrm{mg} / \mathrm{kg}$ every 2 weeks) and pembrolizumab (200 mg, q3w), respectively; these patients were treated until disease progression, intolerable toxicity, or withdrawal by investigator decision, as reported by phase II studies CHECKMATE-205 and KEYNOTE-087, correspondingly $(29,30)$. In another phase II trial ORIENT-4, sintilimab monotherapy showed an anti-tumor effect in extranodal NK/T cell lymphoma (ENKTL) (31). In 28 enrolled patients, sintilimab was given at $200 \mathrm{mg}$ intravenously q3w until disease progression, death, unacceptable toxicity, or withdrawal from the study; the OR rate (ORR) was 67.9\% (19/28); disease control rate (DCR) was $85.7 \%(24 / 28)$ (31). In contrast, pembrolizumab achieved a total ORR of $78.6 \%(11 / 14)$ among 14 ENKTL patients in two retrospective studies $(32,33)$. Although the efficacy of both drugs seems similar, the conclusion about their efficacy on ENKTL may not be rigorous because of the small sample sizes. In addition, sintilimab was also reported to be used in a human immunodeficiency virus-infected $\mathrm{r} / \mathrm{r}$ cHL patient, with a satisfying efficacy and mild, acceptable toxicities (34). In diffuse large B-cell lymphoma, a triple combination of decitabine, sintilimab plus a histone deacetylase inhibitor chidamide achieved partial remission (PR) (35).

\section{Efficacy of Sintilimab in Non-Small Cell Lung Cancer}

In non-small cell lung cancer (NSCLC), sintilimab monotherapy has also proved effective. In a phase Ib study (ChiCTR-OIC17013726), 40 patients with resectable NSCLC were enrolled and given two doses of intravenous sintilimab (200 mg) q3w, followed by an operation; neoadjuvant sintilimab resulted in an ORR of $20.0 \%(8 / 40)$ and a DCR of $90 \%$ (36/40) (36). Among 37 patients undergoing radical resection, 15 (40.5\%) achieved major pathological response (MPR), defined as tumors with $10 \%$ viable tumor cells or less; the 37 patients included six (16.2\%) with a pathologic complete response (pCR) in primary tumor and three (8.1\%) in lymph nodes as well (36-38). In NCT02259621, two preoperative doses of nivolumab $(3 \mathrm{mg} / \mathrm{kg})$ were administered every 2 weeks among 21 patients with stage I, II, or IIIA NSCLC; neoadjuvant nivolumab resulted in an ORR of 9.5\% (2/21) and a DCR of 95.2\% (20/21) among enrolled patients, with 42.9\% (9/ 21) achieving MPR (39). In a phase I study MK3475-223, two doses of pembrolizumab (200 $\mathrm{mg}$ ) was administered in stage I and II NSCLC patients, achieving an MPR of 40\% (4/10) $(40,41)$. Sintilimab seems to have a similar efficacy to nivolumab and pembrolizumab in resectable NSCLC patients when used preoperatively. However, the conclusion may need further evidence in consideration of the small sample sizes.

In addition, sintilimab plus chemotherapy has a strong antitumor activity in NSCLC. A multicenter, phase Ib study involving 20 patients in China (NCT02937116) has shown the safety and well tolerance of sintilimab with a satisfying efficacy (42). In this study, sintilimab $200 \mathrm{mg}$ q3w in combination with gemcitabine and cisplatin regimen was administered in treating naive patients with advanced squamous cell NSCLC until disease progression or intolerant toxicity, yielding a 64.7\% (11/17) ORR, a $30 \%(6 / 17)$ stable disease (SD) proportion among those who have received at least one radiological assessment (42). Most recently, a randomized, double-blind, phase III study (ORIENT11) involving 397 stage IIIB to IV non-squamous NSCLC patients with no previous systemic treatment has shown that the combination of sintilimab and pemetrexed plus platinum has led to significantly longer progression-free survival (PFS) than that of chemotherapy alone with manageable adverse effects (43). In ORIENT-11, patients received either sintilimab $200 \mathrm{mg}$ or placebo plus pemetrexed and platinum $\mathrm{q} 3 \mathrm{w}$ for 4 cycles as induction therapy, followed by sintilimab or placebo plus pemetrexed as maintenance therapy $\mathrm{q} 3 \mathrm{w}$ for up to 24 months; although median overall survival (OS) was not reached, the median PFS, ORR, DCR were 8.9 months, 51.9\% (138/266), $86.8 \%(231 / 266)$ in the sintilimab-combination group compared to those of 5.0 months, $29.8 \%(39 / 131)$ and $75.6 \%(99 / 131)$ in the placebo-combination group (placebo plus chemotherapy) (43). In contrast, in a phase III study KEYNOTE-189, previously untreated patients with metastatic non-squamous NSCLC were also assigned to receive pemetrexed and platinum plus pembrolizumab $(n=410)$ or placebo $(n=206) q 3 w$ for 4 cycles; the median PFS, median OS, ORR, and DCR of patients in the pembrolizumab group were 9.0 and 22.0 months, $48.0 \%$ (197/410), 84.6\% (347/410), compared to those of 4.9 and 10.7 months, $19.4 \%(40 / 266), 70.4 \%(145 / 206)$ of patients in the placebo group (44). By comparison, sintilimab plus chemotherapy has shown similar PFS, ORR, DCR to pembrolizumab and was demonstrated effective.

In single cases, sintilimab was also reported effective in NSCLC. The combination of sintilimab and bevacizumab has led to shrinkage in tumor of a 53-year-old male patient with metastatic adenocarcinoma and resulted in PFS of 6.0 months (45). Also, in ChiCTR-OIC-17013726, one patient with a radiographic assessment of progressive disease (PD) according to RECIST 1.1 was identified with a $60 \%$ pathologic remission at primary tumor site after operation; two patients with radiographic response achieved $100 \%$ pathologic remission at the primary tumor site $(36,46)$. The sign of the PD patient in 
ChiCTR-OIC-17013726 was named pseudoprogression, indicating that the patient who was evaluated as PD according to RECIST 1.1 was actually undergoing late but durable responses (47). In our previous report, pseudoprogression and $100 \%$ pathologic remission were presented in an NSCLC case (48). In that report, a 64-year-old woman diagnosed with initially unresectable squamous NSCLC was given sintilimab plus nedaplatin and paclitaxel for three cycles before surgery; the patient achieved pCR and remained disease-free in the following several-month follow-up (48). In another report, a 65-year-old female patient with unresectable hepatoid adenocarcinoma, docetaxel plus sintilimab has resulted in an SD and a significant PR later on (49). Moreover, sintilimab was reported to maintain effective in a patient more than 3 months after drug withdrawal (50). Because of the satisfying efficacy of sintilimab on NSCLC, the National Medical Products Administration of China has officially accepted the application for the use of sintilimab plus gemcitabine as the first-line therapy in advanced NSCLC $(51,52)$. There may likely be an approval for the use of sintilimab in treating NSCLC in the near future.

\section{Efficacy of Sintilimab in Digestive System Cancers}

In digestive system cancers, sintilimab showed favorable antitumor effects as well. In a phase I study NCT02937116, 20 gastric/gastroesophageal junction adenocarcinoma (G/GEJ) patients were enrolled; among them, $200 \mathrm{mg}$ sintilimab was administered intravenously in combination with CapeOx $(1,000$ $\mathrm{mg} / \mathrm{m}^{2}$ capecitabine orally, bid, days $1-14$ and $130 \mathrm{mg} / \mathrm{m}^{2}$ oxaliplatin intravenously, day 1) every 21 days for up to six cycles; after combination treatment, patients continued to receive sintilimab (200 $\mathrm{mg})$ at 3 weekly intervals as maintenance therapy until progressive disease, unacceptable toxicity, withdrawal of informed consent, or for up to 24 months (53). The combination therapy resulted in PFS of 7.5 months, an ORR of $85 \%$ and a DCR of $100 \%$ (53). In comparison, nivolumab in combination with CapeOx until disease progression, unacceptable toxicity, or consent withdrawal resulted in PFS, ORR, DCR of 10.6 months, 76.5\% (13/17) and $88.2 \%(15 / 17)$ in such patients (54). The combination of CapeOx and sintilimab seems to be superior to that of CapeOx and nivolumab in terms of ORR and DCR although it has shorter PFS. However, the conclusion needs further evidence since the sample sizes are small.

In a phase II study ORIENT-2, 190 advanced esophageal squamous cell carcinoma (ESCC) patients refractory to first-line chemotherapy were randomly assigned (1:1) to receive sintilimab (200 mg, q3w) or chemotherapy (paclitaxel, 175 mg/m2, q3w; or irinotecan, $180 \mathrm{mg} / \mathrm{m} 2$, per 2 weeks), intravenously (55). The median OS was 7.2 versus 6.2 months, and the ORRs were $12.6 \%$ (12/95) versus $6.3 \%$ (6/95) (55). In comparison, ORRs of patients who were refractory to first-line chemotherapy and received pembrolizumab (200 mg q3w) or nivolumab (240 mg every two weeks) alone until disease progression, unacceptable toxic effects, or study withdrawal were $14.3 \%(9 / 63)$ and $19.3 \%(33 / 171)$, respectively; their median OS was 6.8 and 10.9 months $(56,57)$.
The ORR of sintilimab is inferior to nivolumab and pembrolizumab, while its median OS is between them. In a phase Ib study NCT04072679, sintilimab was combined with IBI 305, a biosimilar candidate of bevacizumab, to treat hepatocellular carcinoma (HCC); in that study, sintilimab 200 mg plus IBI $3057.5 \mathrm{mg} / \mathrm{kg}$ or IBI $30515 \mathrm{mg} / \mathrm{kg} \mathrm{q3w}$ was applied in two groups of advanced or metastatic HCC patients, achieving ORRs of $24.1 \%(7 / 29)$ and $33.3 \%(4 / 21)$, respectively (58). However, when combined with fruquintinib, a vascular endothelial growth factor receptor (VEGFR) inhibitor, sintilimab seemed not to have resulted in a significant increase in ORR, DCR, and OS in refractory metastatic colorectal cancer (59).

In single cases, sintilimab has also shown anti-tumor effect in digestive system cancers. In a 46-year-old male patient diagnosed with HCC, lung metastasis was confirmed after resection of the right anterior section of the liver and intraabdominal metastasis (60). Sintilimab and IBI305 were applied and lead to complete remission (CR) (60). Besides, in a 54-year-old female patient with metastatic intrahepatic cholangiocarcinoma (ICC), sintilimab was provided after the failure of the first-line treatment of gemcitabine plus cisplatin chemotherapy; CR was achieved after three cycles of treatment (61). In another case, a 63-year-old woman was diagnosed with stage IV ICC involving multiple sites, PR was achieved after six cycles of combined therapy with sintilimab injection and tegafur-gimeracil-oteracil potassium capsules (62). Sintilimab was also reported to exert anti-tumor effects in metastatic gastric cancer and esophageal cancer, leading to the repression of tumor progression and a decrease in serum tumor markers (63). Considering the reported effect in these cases and ongoing studies such as NCT04072679 (58), digestive system cancers like HCC and ESCC might also be indications of sintilimab in the future.

\section{Efficacy of Sintilimab in Other Tumors}

In other tumors, sintilimab was also reported to be effective. In a prospective study analyzing the efficacy and safety of sintilimab in late stage solid tumors, including melanoma, lung cancer, digestive system caners, and neuroendocrine carcinoma, CR and PR were achieved in two $(3.8 \%, 2 / 53$; one melanoma and one NSCLC) and 21 (39.6\%, 21/53; one neuroendocrine carcinoma, 10 NSCLC and $10 \mathrm{G} / \mathrm{GEJ}$ ) patients, respectively (64). In the study, sintilimab was administered at a dose of $200 \mathrm{mg}$ q3w alone or with first-line chemotherapy until disease progression, intolerable toxicity or withdrawal by investigators' decision (64). The median PFS in this study was 118 (95\% CI, 96-140) days, and median OS was not reached. In another retrospective single center study involving 10 patients with advanced renal cell carcinoma, sintilimab plus axitinib leads to a $40.0 \%$ ORR $(4 / 10)$ and a $90.0 \%$ DCR (9/10) (65). The characteristics of major clinical studies mentioned above are listed in Table 1. In addition, there are ongoing trials of sintilimab on various kinds of cancers, such as those on NSCLC and hepatocellular carcinoma (66). Their results might lead to new indications of this drug in the future. 
TABLE 1 | Summary of major clinical studies of sintilimab.

\begin{tabular}{|c|c|c|c|c|c|c|c|c|c|}
\hline $\begin{array}{l}\text { Clinical } \\
\text { studies }\end{array}$ & ORIENT-1 & ORIENT-2 & ORIENT-4 & ORIENT-11 & $\begin{array}{c}\text { ChicTR-OIC- } \\
17013726\end{array}$ & NCT02937116 & NCT02937116 & NCT02937116 & NA (Jin Liu) \\
\hline Cancer type & $\mathrm{r} / \mathrm{r} \mathrm{cHL}$ & ESCC & ENKTL & NSCLC & NSCLC & ST & NSCLC & G/GEJ & ST \\
\hline $\begin{array}{l}\text { Numbers of } \\
\text { cases }\end{array}$ & 96 & $\begin{array}{l}190 \text { ( } \mathrm{n}=95 \\
\text { per group) }\end{array}$ & 28 & 397 & 40 & 12 & 20 & 20 & 63 \\
\hline $\begin{array}{l}\text { Therapeutic } \\
\text { regimen }\end{array}$ & sintilimab & $\begin{array}{l}\text { sintilimab vs. } \\
\text { chemotherapy }\end{array}$ & sintilimab & $\begin{array}{l}\text { sintilimab plus } \\
\text { chemotherapy } \\
\text { vs. } \\
\text { chemotherapy }\end{array}$ & $\begin{array}{l}\text { sintilimab } \\
\text { (neoadjuvant) }\end{array}$ & sintilimab & $\begin{array}{l}\text { sintilimab plus } \\
\text { chemotherapy }\end{array}$ & $\begin{array}{l}\text { sintilimab plus } \\
\text { chemotherapy }\end{array}$ & $\begin{array}{l}\text { Sintilimab alone (mono) } \\
\text { or plus chemotherapy } \\
\text { (comb) }\end{array}$ \\
\hline ORR & $\begin{array}{l}80.4 \%(74 / \\
92)\end{array}$ & 12.6 vs. $6.3 \%$ & $\begin{array}{l}68 \%(19 / \\
28)\end{array}$ & $\begin{array}{l}51.9 \%(138 / \\
266) v s . \\
29.8 \% \text { (39/ } \\
131)\end{array}$ & $20.0 \%(8 / 40)$ & $18.2 \%(2 / 11)$ & $64.7 \%(11 / 17)$ & $85.0 \%(17 / 20)$ & $\begin{array}{l}18.5 \% \text { (5/27, mono), } \\
69.2 \% \text { (18/26, comb) }\end{array}$ \\
\hline DCR & $\begin{array}{l}97.8 \%(90 / \\
92)\end{array}$ & NA & $\begin{array}{l}85.7 \%(24 / \\
28)\end{array}$ & $\begin{array}{l}86.8 \%(231 / \\
266) v s . \\
75.6 \%(99 / \\
131)\end{array}$ & $90.0 \%(36 / 40)$ & $36.4 \%(4 / 11)$ & $100 \%(17 / 17)$ & $100 \%(20 / 20)$ & $\begin{array}{l}66.7 \%(18 / 27, \text { mono)} \\
96.2 \%(25 / 26, \mathrm{comb})\end{array}$ \\
\hline TRAES & $\begin{array}{l}54.0 \%(52 / \\
96)\end{array}$ & $\begin{array}{l}54.3 \text { vs. } \\
90.8 \%\end{array}$ & $\begin{array}{l}100 \%(28 / \\
28)\end{array}$ & $\begin{array}{l}99.6 \%(265 / \\
266) \text { vs. } 100 \% \\
(131 / 131)\end{array}$ & $52.5 \%(21 / 40)$ & NA & $100 \%(20 / 20)$ & $100 \%(20 / 20)$ & $79.3 \%(50 / 63)$ \\
\hline $\begin{array}{l}\text { The most } \\
\text { common } \\
\text { TRAEs }\end{array}$ & $\begin{array}{l}\text { Pyrexia } \\
(38 \%, 36 / \\
96)\end{array}$ & NA & NA & $\begin{array}{l}\text { Anemia } \\
\text { (74.1\%, 197/ } \\
266 \text { vs. } \\
78.6 \%, 103 / \\
131)\end{array}$ & $\begin{array}{l}\text { Asthenia } \\
(17.5 \%, 7 / 40) \\
\text { hypothyroidism } \\
(17.5 \%, 7 / 40)\end{array}$ & $\begin{array}{l}\text { Fever, thyroid } \\
\text { dysfunction, } \\
\text { serum bilirubin } \\
\text { elevation and } \\
\text { pneumonitis } \\
\text { (25.0\%, 3/12 } \\
\text { each) }\end{array}$ & $\begin{array}{l}\text { Rash }(15.0 \% \text {, } \\
3 / 20)\end{array}$ & $\begin{array}{l}\text { Decreased } \\
\text { platelet count } \\
(80 \%, 16 / 20)\end{array}$ & $\begin{array}{l}\text { Altered } \\
\text { electrocardiogram } \\
\text { (32.3\%, 10/31, all grade } \\
\text { 1-2, mono), hematologic } \\
\text { abnormalities (65.6\%, } \\
\text { 21/32, comb) }\end{array}$ \\
\hline $\begin{array}{l}\text { Grade } 1-2 \\
\text { TRAEs }\end{array}$ & $\begin{array}{l}51.0 \%(49 / \\
96)\end{array}$ & $\begin{array}{l}34.1 \mathrm{vs} . \\
51.7 \%\end{array}$ & $\begin{array}{l}60.7 \%(17 / \\
28)\end{array}$ & $\begin{array}{l}38.0 \%(101 / \\
266) v s . \\
41.2 \%(54 / \\
131)\end{array}$ & $42.5 \%(17 / 40)$ & NA & $30.0 \%(6 / 20)$ & $45 \%(9 / 20)$ & $65.1 \%(41 / 63)$ \\
\hline $\begin{array}{l}\text { Grade 3-4 } \\
\text { TRAEs }\end{array}$ & $\begin{array}{l}3.1 \%(3 / \\
96)\end{array}$ & NA & $\begin{array}{l}39.3 \%(11 / \\
28)\end{array}$ & $\begin{array}{l}59.4 \%(158 / \\
266) v s . \\
51.9 \%(68 / \\
131)\end{array}$ & $7.5 \%(3 / 40)$ & $33.3 \%(4 / 12)$ & $70.0 \%(14 / 20)$ & $55 \%(11 / 20)$ & $\begin{array}{l}16.1 \% \text { (5/31, mono), } \\
12.5 \% \text { (4/32, comb) }\end{array}$ \\
\hline $\begin{array}{l}\text { Grade } 5 \\
\text { TRAEs }\end{array}$ & 0 & NA & 0 & $\begin{array}{l}2.3 \%(6 / 266) \\
\text { vs. } 6.9 \%(9 / \\
131)\end{array}$ & $2.5 \%(1 / 40)$ & 0 & 0 & 0 & 0 \\
\hline
\end{tabular}

In some studies, numbers of assessable patients were less than the numbers of cases.

NA, not available/applicable; r/r cHL, relapsed or refractory classical Hodgkin lymphoma; ESCC, esophageal squamous cell carcinoma; ENKTL, extranodal NK/T cell lymphoma; ST, solid tumors; NSCLC, non-small cell lung cancer; G/GEJ, gastric/gastroesophageal junction adenocarcinoma; vs., versus; ORR, overall survival rate; DCR, disease control rate; TRAE, treatment-related adverse effect; ALT, alanine transaminase; AST, aspartate transaminase.

\section{PREDICTIVE PARAMETERS OF SINTILIMAB EFFICACY}

In the above-mentioned studies, some indexes were shown to be predictive of sintilimab efficacy. In a biomarker study involving 75 participants from ORIENT-1, circulating tumor DNA (ctDNA) was proved to be a potential biomarker for sintilimab immunotherapy (5). Among patients with a ctDNA, a decrease of $\geq 40$ and $79.31 \%(23 / 29)$ had an agreement between the change in ctDNA and the best radiographic response, with CR and PR achieved in 90\% (5). Also, patients whose tumor area decrease is equal or larger than $60 \%$ exhibited a more substantial ctDNA decline than those whose tumor area decrease is less than 60\% (5). In ChiCTR-OIC17013726, squamous cell NSCLC exhibited a better pathological response to sintilimab than did adenocarcinoma (MPR: 48.4 versus 0\%) (36). Moreover, a significant correlation was observed between maximum standardized uptake values (SUVmax) reduction and pathologic response (36). In 2009, traditional SUV was replaced by standard uptake value of lean body mass (SUL) in the PERCIST 1.0 standard which was proposed for evaluating the efficacy of solid tumors with positron emission tomography (PET)-computer tomography (CT) (67). Thus, the correlation between SULmax, a replacement of SUVmax, and the pathological response of 36 participants in ChiCTR-OIC-17013726 was analyzed in another study by Tao et al. (68). The study demonstrated that SULmax of PET-CT performed at baseline was positively correlated with the degree of pathological regression of primary tumor; the study also showed that all metabolic parameters of PET-CT performed within 1 week prior to surgery and the percentage changes of metabolic parameters after neoadjuvant therapy were negatively correlated with the regression of tumor (68). 


\section{ADVERSE EFFECTS OF SINTILIMAB}

Although sintilimab has shown a potent efficacy in the treatment of tumors, its side effects and its potential damage to patients are inevitable. In the official instructions, the adverse effects of sintilimab included pneumonia, diarrhea, colitis, hepatitis, nephritis, endocrinology diseases, skin AEs, infusion reactions, and other immune-related AEs (27). The AEs were defined according to the Common Terminology Criteria for Adverse Events (CTCAEs) from the National Cancer Institute and were coded using the Medical Dictionary for Regulatory Activities $(69,70)$. The CTCAE have five grades, referring to mild, moderate, severe, life-threatening AEs or death, in an ascending order.

As listed in Table 1, treatment-related AEs (TRAEs) were reported in most of the patients in major clinical studies and the incidences range from 52.5 to $100 \%$ among them. In ORIENT-1, TRAEs occurred in $92.7 \%(89 / 96)$ patients, and grade 3-5 TRAEs occurred in $17.7 \%$ (17/96) patients, without TARErelated death (26). In CHECKMATE-205, TRAEs occurred in 98.8\% (79/80) patients, grade 3-5 TRAEs occurred in 41.3\% (32/ 80) patients, and there was one TARE-related death (29). In KEYNOTE-087, no patient died of TRAEs although the occurrence rate of TRAEs and grade 3-5 TRAEs were not available (30). Thus, sintilimab caused less toxicities in patients with $\mathrm{r} / \mathrm{r} \mathrm{cHL}$ than nivolumab and was proved safe. In ChiCTROIC-17013726, the incidence of all grade TRAEs was 52.5\% (21/ 40); the incidence of 3-5 TRAEs was $10.0 \%(4 / 40)$, and the rate of TRAE-related death was 2.5\% (1/40) (36). In NCT02259621, the incidences and rate were $22.7 \%(5 / 22), 4.5 \%(1 / 22)$, and 0 (39). In MK3475-223, the data were not available (40, 41). Although the safety profile of sintilimab in NSCLC seems worse than nivolumab when used preoperatively, the conclusion needs more evidence since the sample size of the latter is small.

The safety of ICIs has been evaluated in a previous study revealing an incidence rate varying between 54 and $76 \%$ for all AEs (71). When sintilimab was used alone, TRAEs occurred in more than $58.0 \%$ (120/207) patients, with 13.0\% (27/207) grade 3 or higher TRAEs and one death $(0.5 \%, 1 / 207)(25,26,36,64,72)$. For nivolumab, a pooled analysis of four major clinical trials on NSCLC showed that $69.4 \%$ (461/664) of patients had TRAEs and that grade 3-5 treatment-related adverse events occurred in $13.9 \%(92 / 664)$ of patients; overall, there are $0.9 \%(6 / 664)$ deaths (73). For pembrolizumab in NSCLC, the rate of TRAEs was $66.3 \%$ (1304/1967), with $15.2 \%$ (299/1967) grade 3 or higher TRAEs when used alone, and at least 1.0\% (20/1967) deaths (7477). Sintilimab seems safer than nivolumab or pembrolizumab when used alone because of its lower TRAE incidence and death rate. However, the conclusion may not be accurate because the studies of sintilimab involve various cancers in addition to NSCLC.

When sintilimab was combined with chemotherapy (pemetrexed and platinum), TRAEs occurred in 99.6\% (265/ 266) of patients, with $61.7 \%(164 / 266)$ grade 3 or higher TRAEs and $2.3 \%(6 / 266)$ of AE-related deaths compared to those of
$100 \%$ (131/131), $58.8 \%$ (77/131), and 6.9\% (9/131) in the control group (43). When pembrolizumab was combined with pemetrexed and platinum, the rate of TRAEs reached $99.8 \%$ (404/405), with $71.9 \%(291 / 405)$ grade 3 or higher TRAEs, and 29 deaths $(7.2 \%, 29 / 405)$ (44). Preliminarily, when combined with pemetrexed and platinum, the incidences of TRAEs are similar but the incidence of $\geq 3$ TRAEs and the death rate are lower for sintilimab. Thus, sintilimab had better safety profiles compared to nivolumab or pembrolizumab when used alone in $\mathrm{r} / \mathrm{r} \mathrm{cHL}$ and when combined with pemetrexed and platinum in advanced NSCLC.

Besides, there were also AEs not mentioned in clinical studies or in the drug instructions. In the study by Xing et al., a 66-yearold lung adenocarcinoma patient received two doses of sintilimab as monotherapy and developed myositis-myasthenia gravis overlap syndrome complicated with myasthenia crisis; the overlap syndrome was cured after supportive therapies such as plasma exchange, mechanical ventilation, and immunosuppressive therapy (78). In an HCC patient, sintilimab administration resulted in autoimmune diabetes at 24 weeks after injection (79). In other reported cases, patients developed cytokine release syndrome, pulmonary fibrosis, hypothyroid myopathy, encephalitis during or after sintilimab administration (80-83). All syndromes in these patients were relieved after drug withdrawal and glucocorticoid treatment; there were no deaths in these cases.

\section{DISCUSSION}

Sintilimab is a domestic PD-1 antibody in China and has received its approval on 24 December 2018 for the treatment of $\mathrm{r} / \mathrm{r} \mathrm{cHL}$. The drug has shown promising anti-tumor effect on various cancer types in a variety of studies. The studies related to sintilimab were summarized in a timeline in Figure 1. The results of these studies have indicated a bright future for sintilimab.

However, there are obvious limitations. First of all, there is lack of direct head-to-head comparisons to other PD-1 inhibitors like nivolumab and pembrolizumab. Secondly, among the studies included, complete data of some studies, such as ORIENT-2 and ORIENT-4, are not available. More importantly, almost all of the patients included are native Chinese, and there is only one registered study outside China (NCT03748134/ORIENT-15) (66). The efficacy of this drug remains to be explored in people of other races. Future studies addressing these questions could be implemented.

Despite the limitations, there are some aspects worth expecting. First, there are a number of ongoing clinical studies assessing sintilimab for treating various cancers (66). In addition, in a health technology assessment, sintilimab got a total score of 68, compared to those of 75 for nivolumab, 62 for pembrolizumab, 66 for toripalimab, and 58 for camrelizumab (84). Furthermore, sintilimab has a lower cost (approximately 24,000 USD/year) than other well-studied PD-1 inhibitors (approximately 87,000 USD/year for pembrolizumab and 


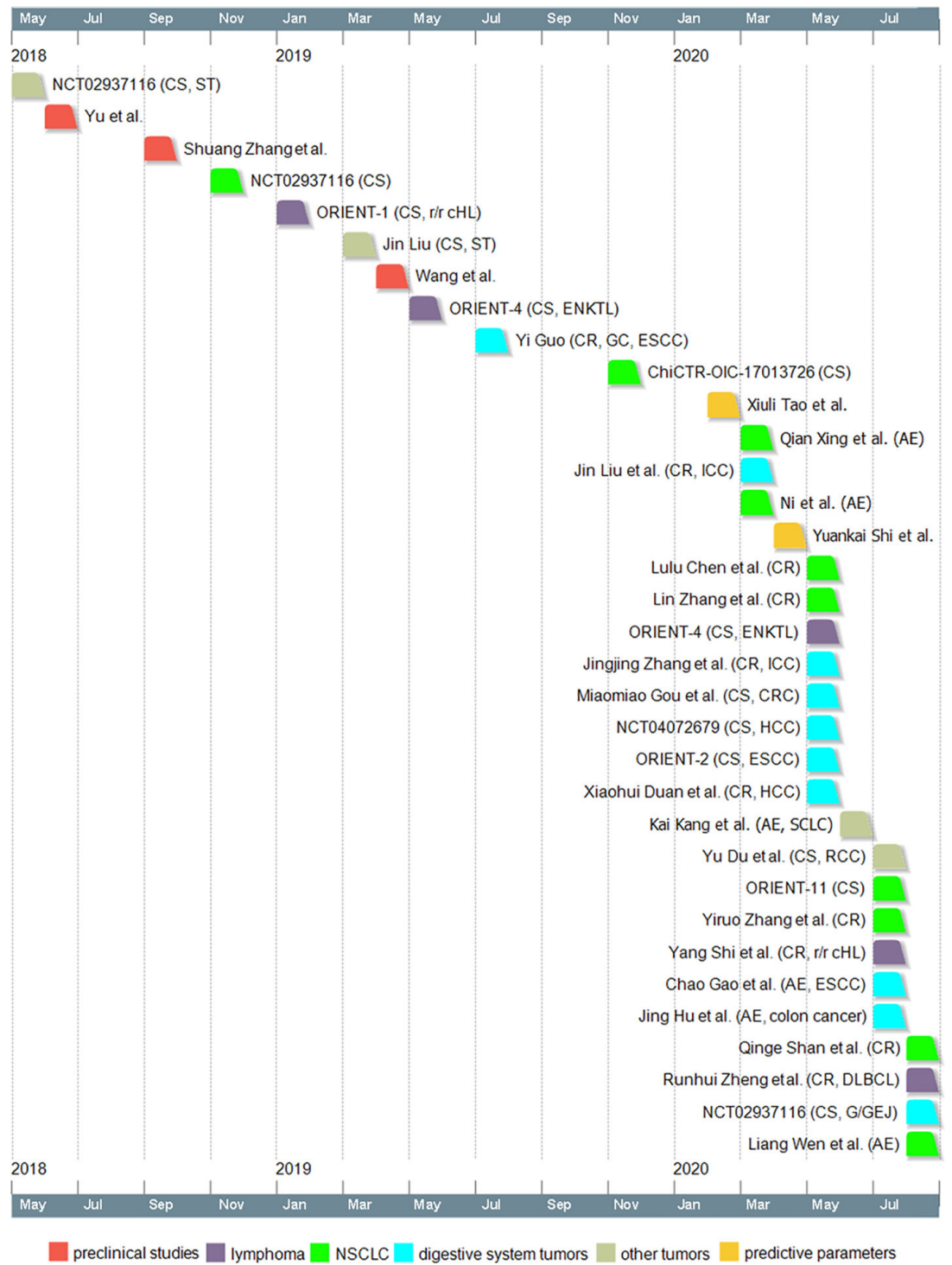

FIGURE 1 | Timeline of sintilimab-related studies. Dates indicate the time when the study was first available online. CS, clinical study; ST, solid tumors; r/r cHL, relapsed or refractory classical Hodgkin lymphoma; ENKTL, extranodal NK/T cell lymphoma; CR, case report; GC, gastric cancer; ESCC, esophageal squamous cell carcinoma; AE, case reports on adverse effects; ICC, intrahepatic cholangiocarcinoma; CRC, colorectal cancer; HCC, hepatocellular carcinoma; SCLC, small cell lung cancer; RCC, renal cell carcinoma; DLBCL, diffuse large B-cell lymphoma; G/GEJ, gastric/gastroesophageal junction adenocarcinoma; NSCLC, non-small cell lung cancer.

63,000 USD/year for nivolumab). Considering the different economic background of Chinese patients from that of patients in developed western countries, the lower cost of sintilimab can sometimes be decisive in their choices of cancer treatment.

\section{CONCLUSION}

In conclusion, sintilimab has been preliminarily proved effective and safe in the treatment of various cancers. Although the efficacy of sintilimab in these cancers still needs to be validated in further research, it is hopeful that sintilimab will be approved with more indications in the near future. Our review has provided clinical physicians with a potential choice for the treatment of cancers in the future.

\section{AUTHOR CONTRIBUTIONS}

LZ: conceptualization. LZ and WM: writing, visulization, and original draft. WJ and QG: review and editing. QG: funding. All authors contributed to the article and approved the submitted version. 


\section{FUNDING}

This work was supported by National Natural Science Foundation of China granted to QG (81770095).

\section{REFERENCES}

1. Edner NM, Carlesso G, Rush JS, Walker LSK. Targeting co-stimulatory molecules in autoimmune disease. Nat Rev Drug Discovery (2020). doi: 10.1038/s41573-020-0081-9

2. Marin-Acevedo JA, Dholaria B, Soyano AE, Knutson KL, Chumsri S, Lou Y. Next generation of immune checkpoint therapy in cancer: new developments and challenges. J Hematol Oncol (2018) 11:39. doi: 10.1186/s13045-018-0582-8

3. $\mathrm{He} \mathrm{X}, \mathrm{Xu} \mathrm{C}$. Immune checkpoint signaling and cancer immunotherapy. Cell Res (2020) 30:660-9. doi: 10.1038/s41422-020-0343-4

4. Hoy SM. Sintilimab: First Global Approval. Drugs (2019) 79:341-6. doi: $10.1007 / \mathrm{s} 40265-019-1066-z$

5. Shi Y, Su H, Song Y, Jiang W, Sun X, Qian W, et al. Circulating tumor DNA predicts response in Chinese patients with relapsed or refractory classical hodgkin lymphoma treated with sintilimab. EBioMedicine (2020) 54:102731. doi: 10.1016/j.ebiom.2020.102731

6. Ishida Y, Agata Y, Shibahara K, Honjo T. Induced expression of PD-1, a novel member of the immunoglobulin gene superfamily, upon programmed cell death. EMBO J (1992) 11:3887-95. doi: 10.1002/j.1460-2075.1992.tb05481.x

7. Kong X. Discovery of New Immune Checkpoints: Family Grows Up. Adv Exp Med Biol (2020) 1248:61-82. doi: 10.1007/978-981-15-3266-5_4

8. Wang J, Yang T, Xu J. Therapeutic Development of Immune Checkpoint Inhibitors. Adv Exp Med Biol (2020) 1248:619-49. doi: 10.1007/978-981-153266-5_23

9. Okazaki T, Maeda A, Nishimura H, Kurosaki T, Honjo T. PD-1 immunoreceptor inhibits B cell receptor-mediated signaling by recruiting src homology 2-domaincontaining tyrosine phosphatase 2 to phosphotyrosine. Proc Natl Acad Sci USA (2001) 98:13866-71. doi: 10.1073/pnas.231486598

10. Chemnitz JM, Parry RV, Nichols KE, June CH, Riley JL. SHP-1 and SHP-2 associate with immunoreceptor tyrosine-based switch motif of programmed death 1 upon primary human $\mathrm{T}$ cell stimulation, but only receptor ligation prevents T cell activation. J Immunol (2004) 173:945-54. doi: 10.4049/ jimmunol.173.2.945

11. Ribas A, Wolchok JD. Cancer immunotherapy using checkpoint blockade. Science (2018) 359:1350-5. doi: 10.1126/science.aar4060

12. Homet Moreno B, Ribas A. Anti-programmed cell death protein-1/ligand-1 therapy in different cancers. Brit J Cancer (2015) 112:1421-7. doi: 10.1038/ bjc. 2015.124

13. Francisco LM, Sage PT, Sharpe AH. The PD-1 pathway in tolerance and autoimmunity. Immunol Rev (2010) 236:219-42. doi: 10.1146/ annurev.immunol.26.021607.090331

14. Patsoukis N, Wang Q, Strauss L, Boussiotis VA. Revisiting the PD-1 pathway. Sci Adv (2020) 6:eabd2712. doi: 10.1126/sciadv.abd2712

15. Chen L, Han X. Anti-PD-1/PD-L1 therapy of human cancer: past, present, and future. J Clin Invest (2015) 125:3384-91. doi: 10.1172/JCI80011

16. Zou W, Wolchok JD, Chen L. PD-L1 (B7-H1) and PD-1 pathway blockade for cancer therapy: Mechanisms, response biomarkers, and combinations. Sci Transl Med (2016) 8:324r-8r. doi: 10.1126/scitranslmed.aad7118

17. Azuma T, Yao S, Zhu G, Flies AS, Flies SJ, Chen L. B7-H1 is a ubiquitous antiapoptotic receptor on cancer cells. Blood (2008) 111:3635-43. doi: 10.1182/blood-2007-11-123141

18. Wu Y, Gu W, Li J, Chen C, Xu ZP. Silencing PD-1 and PD-L1 with nanoparticle-delivered small interfering RNA increases cytotoxicity of tumor-infiltrating lymphocytes. Nanomedicine(Lond) (2019) 14:955-67. doi: $10.2217 / \mathrm{nnm}-2018-0237$

19. Kleffel S, Posch C, Barthel SR, Mueller H, Schlapbach C, Guenova E, et al. Melanoma Cell-Intrinsic PD-1 Receptor Functions Promote Tumor Growth. Cell (2015) 162:1242-56. doi: 10.1016/j.cell.2015.08.052

20. Wang J, Fei K, Jing H, Wu Z, Wu W, Zhou S, et al. Durable blockade of PD-1 signaling links preclinical efficacy of sintilimab to its clinical benefit. MAbs (2019) 11:1443-51. doi: 10.1080/19420862.2019.1654303

\section{ACKNOWLEDGMENTS}

We thank editors and reviewers for their helpful suggestions to this work.

21. Zhang S, Zhang M, Wu W, Yuan Z, Tsun A, Wu M, et al. Preclinical characterization of Sintilimab, a fully human anti-PD-1 therapeutic monoclonal antibody for cancer. Antib Ther (2018) 1:65-73. doi: 10.1093/ abt/tby005

22. Keir ME, Butte MJ, Freeman GJ, Sharpe AH. PD-1 and its ligands in tolerance and immunity. Annu Rev Immunol (2008) 26:677-704. doi: 10.1146/ annurev.immunol.26.021607.090331

23. van der Neut Kolfschoten M, Schuurman J, Losen M, Bleeker WK, MartinezMartinez P, Vermeulen E, et al. Anti-Inflammatory Activity of Human IgG4 Antibodies by Dynamic Fab Arm Exchange. Science (2007) 317:1554-7. doi: $10.1126 /$ science.1144603

24. Chuanfei Y, Xingjun C, Wenbo W, Haiqing N, GUO AY, Lan W. [LC-MS analysis of sintilimab as an anti-PD-1 therapeutic mab for its improved hinge stability study](in Chinese). Acta Pharmaceu Sin (2019) 54:122-9. doi: 10.16438/j.0513-4870.2018-0574

25. Xu J, Jia R, Wang Y, Liu R, Zhao C, Zhou H, et al. A first-in-human phase 1a trial of sintilimab (IBI308), a monoclonal antibody targeting programmed death-1 (PD-1), in Chinese patients with advanced solid tumors. J Clin Oncol (2018) 36:e15125. doi: 10.1200/JCO.2018.36.15_suppl.e15125

26. Shi Y, Su H, Song Y, Jiang W, Sun X, Qian W, et al. Safety and activity of sintilimab in patients with relapsed or refractory classical Hodgkin lymphoma (ORIENT-1): a multicentre, single-arm, phase 2 trial. Lancet Haematol (2019) 6:e12-9. doi: 10.1016/S2352-3026(18)30192-3

27. Instructions for sintilimab injection (2020). Available at: http://inn-qc. iqiaomai.com/5c89f09e539a2 (Accessed October 4th).

28. Cheson BD, Pfistner B, Juweid ME, Gascoyne RD, Specht L, Horning SJ, et al. Revised response criteria for malignant lymphoma. J Clin Oncol (2007) 25:579-86. doi: 10.1200/JCO.2006.09.2403

29. Younes A, Santoro A, Shipp M, Zinzani PL, Timmerman JM, Ansell S, et al. Nivolumab for classical Hodgkin's lymphoma after failure of both autologous stem-cell transplantation and brentuximab vedotin: a multicentre, multicohort, single-arm phase 2 trial. Lancet Oncol (2016) 17:1283-94. doi: 10.1016/S1470-2045(16)30167-X

30. Chen R, Zinzani PL, Fanale MA, Armand P, Johnson NA, Brice P, et al. Phase II Study of the Efficacy and Safety of Pembrolizumab for Relapsed/Refractory Classic Hodgkin Lymphoma. J Clin Oncol (2017) 35:2125-32. doi: 10.1200/ JCO.2016.72.1316

31. Tao R, Fan L, Song Y, Hu Y, Zhang W, Wang Y, et al. Sintilimab for relapsed/ refractory $(\mathrm{r} / \mathrm{r})$ extranodal NK/T-cell lymphoma (ENKTL): A multicenter, single-arm, phase 2 trial (ORIENT-4). J Clin Oncol (2019) 37S:102-3. doi: $10.1002 /$ hon.66_2629

32. Li X, Cheng Y, Zhang M, Yan J, Li L, Fu X, et al. Activity of pembrolizumab in relapsed/refractory NK/T-cell lymphoma. J Hematol Oncol (2018) 11:15. doi: 10.1186/s13045-018-0559-7

33. Kwong YL, Chan T, Tan D, Kim SJ, Poon LM, Mow B, et al. PD1 blockade with pembrolizumab is highly effective in relapsed or refractory NK/T-cell lymphoma failing l-asparaginase. Blood (2017) 129:2437-42. doi: 10.1182/ blood-2016-12-756841

34. Shi Y, Li Q, Zhang W, Nan Y, Yang T, Liang X, et al. Sintilimab as salvage treatment in an HIV patient with relapsed/ refractory Hodgkin: a case report. Ann Palliat Med (2020) 9:2414-9. doi: 10.21037/apm-20-1333

35. Zheng R, Chen X, Wang C, Qin P, Tan H, Luo X. Triplet Therapy with PD-1 Blockade, Histone Deacetylase Inhibitor, and DNA Methyltransferase Inhibitor Achieves Radiological Response in Refractory Double-Expressor Diffuse Large B-cell Lymphoma with 17p Deletion. Case Rep Hematol (2020) 2020:1-6. doi: 10.1155/2020/8879448

36. Gao S, Li N, Gao S, Xue Q, Ying J, Wang S, et al. Neoadjuvant PD-1 inhibitor (Sintilimab) in NSCLC. J Thorac Oncol (2020) 15:816-26. doi: 10.1016/ j.jtho.2020.01.017

37. Pataer A, Kalhor N, Correa AM, Raso MG, Erasmus JJ, Kim ES, et al. Histopathologic Response Criteria Predict Survival of Patients with 
Resected Lung Cancer After Neoadjuvant Chemotherapy. J Thorac Oncol (2012) 7:825-32. doi: 10.1097/JTO.0b013e318247504a

38. Hellmann MD, Chaft JE, William WN, Rusch V, Pisters KMW, Kalhor N, et al. Pathological response after neoadjuvant chemotherapy in resectable non-small-cell lung cancers: proposal for the use of major pathological response as a surrogate endpoint. Lancet Oncol (2014) 15:e42-50. doi: 10.1016/S1470-2045(13)70334-6

39. Forde PM, Chaft JE, Smith KN, Anagnostou V, Cottrell TR, Hellmann MD, et al. Neoadjuvant PD-1 Blockade in Resectable Lung Cancer. N Engl J Med (2018) 378:1976-86. doi: 10.1007/s00259-020-04711-3

40. Bar J, Urban D, Ofek E, Ackerstein A, Redinsky I, Golan N, et al. Neoadjuvant pembrolizumab (Pembro) for early stage non-small cell lung cancer (NSCLC): Updated report of a phase I study, MK3475-223. J Clin Oncol (2019) 37:8534. doi: 10.1200/JCO.2019.37.15_suppl.8534

41. Ben Nun A, Golan N, Ofek E, Urban D, Kamer I, Simansky D, et al. Neoadjuvant pembrolizumab (Pembro) for early stage non-small cell lung cancer (NSCLC): Initial report of a phase I study, MK3475-223. Ann Oncol (2018) 29 Suppl 8:1486. doi: 10.1093/annonc/mdy290.011

42. Ying $\mathrm{K}, \mathrm{Xu} \mathrm{N}$, Jiang H, Liu Y, Zhou H, Wang S. Efficacy and Safety of Sintilimab Combined with 1st Line Chemotherapy in Advanced Squamous Cell Non-small Cell Lung Cancer. J Thorac Oncol (2018) 13S:S1047. doi: 10.1016/j.jtho.2018.10.018

43. Yang Y, Wang Z, Fang J, Yu Q, Han B, Cang S, et al. Efficacy and safety of sintilimab plus pemetrexed and platinum as first-line treatment for locally advanced or metastatic nonsquamous non-small cell lung cancer: a randomized, double-blind, phase 3 study (ORIENT-11). J Thorac Oncol (2020) 15:1636-46. doi: 10.1016/j.jtho.2020.07.014

44. Gadgeel S, Rodríguez-Abreu D, Speranza G, Esteban E, Felip E, Dómine M, et al. Updated Analysis From KEYNOTE-189: Pembrolizumab or Placebo Plus Pemetrexed and Platinum for Previously Untreated Metastatic Nonsquamous Non-Small-Cell Lung Cancer. J Clin Oncol (2020) 38:150517. doi: $10.1200 / J C O .19 .03136$

45. Zhang Y, Zhao M, Cao S, Zhang X, Du Y. Unexpected favorable outcome to sintilimab plus bevacizumab in an EGFR -mutated non-small cell lung cancer patient: A case report. Thorac Cancer (2020) 11:2717-22. doi: 10.1111/17597714.13569

46. Eisenhauer EA, Therasse P, Bogaerts J, Schwartz LH, Sargent D, Ford R, et al. New response evaluation criteria in solid tumours: revised RECIST guideline (version 1.1). Eur J Cancer (2009) 45:228-47. doi: 10.1016/j.ejca.2008.10.026

47. Seymour L, Bogaerts J, Perrone A, Ford R, Schwartz LH, Mandrekar S, et al. iRECIST: guidelines for response criteria for use in trials testing immunotherapeutics. Lancet Oncol (2017) 18:e143-52. doi: 10.1016/S14702045(17)30074-8

48. Zhang L, Mai W, Hao B, Jiang W, Geng Q. Promising response to a PD-1 inhibitor (sintilimab) in non-small cell lung cancer. Med (Baltimore) (2020) 99:e19790. doi: 10.1097/MD.0000000000019790

49. Chen L, Han X, Gao Y, Zhao Q, Wang Y, Jiang Y, et al. Anti-PD-1 Therapy Achieved Disease Control After Multiline Chemotherapy in Unresectable KRAS-Positive Hepatoid Lung Adenocarcinoma: A Case Report and Literature Review. Onco Targets Ther (2020) 13:4359-64. doi: 10.2147/ OTT.S248226

50. Shan Q, Wang H, Han X, Guo J, Wang Z. Duration of immunotherapy in patients with advanced lung adenocarcinoma with negative driver genes: case report and literature review. Thorac Cancer (2020) 11:3001-6. doi: 10.1111/ 1759-7714.13600

51. China National Medical Products Administration has accepted the application for a new indication of Tyvyt ${ }^{\circledR}$ (Sintilimab injection) to be used as a first-line therapy in combination with gemcitabine for the treatment of squamous non-small cell lung cancer. Available at: http://innoventbio.com/ \#/news/218 (Accessed August 13, 2020). (in Chinese).

52. Inquiry of Drug Registration Progress. Available at: http://sq.nmpa.gov.cn/ datasearch_nmpa/schedule/search.jsp?tableId $=43 \&$ tableName $=$ TABLE43\&columnName $=$ COLUMN464,COLUMN475\&title=\%D2\%A9\% C6\%B7\%D7\%A2\%B2\%E1\%BD\%F8\%B6\%C8\%B2\%E9\%D1\%AF (Accessed August 13, 2020). (in Chinese).

53. Jiang H, Zheng Y, Qian J, Mao C, Xu X, Li N, et al. Safety and efficacy of sintilimab combined with oxaliplatin/capecitabine as first-line treatment in patients with locally advanced or metastatic gastric/gastroesophageal junction adenocarcinoma in a phase Ib clinical trial. BMC Cancer (2020) $20: 760$. doi: $10.1186 / \mathrm{s} 12885-020-07251-\mathrm{z}$

54. Boku N, Ryu MH, Kato K, Chung HC, Minashi K, Lee KW, et al. Safety and efficacy of nivolumab in combination with S-1/capecitabine plus oxaliplatin in patients with previously untreated, unresectable, advanced, or recurrent gastric/gastroesophageal junction cancer: interim results of a randomized, phase II trial (ATTRACTION-4). Ann Oncol (2019) 30:250-8. doi: 10.1093/ annonc/mdy540

55. Xu J, Li Y, Fan Q, Shu Y, Wu Z, Cui T, et al. Sintilimab in patients with advanced esophageal squamous cell carcinoma refractory to previous chemotherapy: A randomized, open-label phase II trial (ORIENT-2). J Clin Oncol (2020) 38:4511. doi: 10.1200/JCO.2020.38.15_suppl.4511

56. Shah MA, Kojima T, Hochhauser D, Enzinger P, Raimbourg J, Hollebecque A, et al. Efficacy and Safety of Pembrolizumab for Heavily Pretreated Patients With Advanced, Metastatic Adenocarcinoma or Squamous Cell Carcinoma of the Esophagus. JAMA Oncol (2019) 5:546. doi: 10.1001/jamaoncol.2018.5441

57. Kato K, Cho BC, Takahashi M, Okada M, Lin C, Chin K, et al. Nivolumab versus chemotherapy in patients with advanced oesophageal squamous cell carcinoma refractory or intolerant to previous chemotherapy (ATTRACTION-3): a multicentre, randomised, open-label, phase 3 trial. Lancet Oncol (2019) 20:1506-17. doi: 10.1016/S1470-2045(19)30626-6

58. Zhang W, Bi X, Sun Y, Yu Y, Zhou J, Zeng H, et al. Preliminary results of sintilimab plus different dose of IBI305 (anti-VEGF monoclonal antibody) in patients with advanced hepatocellular carcinoma: A phase Ib study. J Clin Oncol (2020) 38:3079. doi: 10.1200/JCO.2020.38.15_suppl.3079

59. Gou M, Yan H, Liu Tie E, Wang Z, Si H, Chen S, et al. Fruquintinib combination with sintilimab in refractory metastatic colorectal cancer patients in China. J Clin Oncol (2020) 38:4028. doi: 10.1200/JCO.2020.38.15_suppl.4028

60. Duan X, Zhang H, Zhou L, Jiang B, Mao X. Complete response to the combination of sintilimab and IBI305 for a patient with HBV-associated hepatocellular carcinoma with multiple lung metastasis. Digest Liver Dis (2020) 52:794-6. doi: 10.1016/j.dld.2020.04.005

61. Zhang J, Wu L, Liu J, Lin M. A metastatic intrahepatic cholangiocarcinoma treated with programmed cell death 1 inhibitor: a case report and literature review. Immunotherapy (2020) 12:555-61. doi: 10.2217/imt-2019-0100

62. Liu J, Wang C, Cao L, Li S. Treatment of advanced intrahepatic cholangiocarcinoma with sintilimab combined with tegafur-gimeraciloteracil potassium capsules (S-1): a case report. Ann Palliat Med (2020) 9:497-503. doi: 10.21037/apm.2020.03.14

63. Yi G. [Treatment of Advanced Digestive Tract Tumor with PD-1 Inhibitor in 2 Cases and Literature Review](in Chinese). Shandong Univ (2019) 45. doi: $10.27272 /$ d.cnki.gshdu.2019.000927

64. Jin L. [Efficacay and safety of anti-PD-1 monoclonal antibody in the treatment of advanced malignant solid tumors](in Chinese). Zhejiang Univ (2019) 71. doi: 10.27461/d.cnki.gzjdx.2019.001722

65. Du Y, Si L, Mao L, Chi Z, Cui C, Guo J. [Efficacy of axitinib plus sintilimab in intermediate-and high-risk advanced renal cell carcinoma](in Chinese). Chin J Clin Oncol (2020) 47:513-6. doi: 10.3969/j.issn.1000-8179.2020.10.342

66. Clinical trials on sintilimab. Available at: https://clinicaltrials.gov/ct2/results?cond= \&term $=$ sintilimab\&cntry $=\& s t a t e=\& c i t y=\&$ dist $($ Accessed October 4 th, 2020).

67. Wahl RL, Jacene H, Kasamon Y, Lodge MA. From RECIST to PERCIST: Evolving Considerations for PET Response Criteria in Solid Tumors. J Nucl Med (1978) (2009) 50:122S-50S. doi: 10.2967/jnumed.108.057307

68. Tao X, Li N, Wu N, He J, Ying J, Gao S, et al. The efficiency of 18F-FDG PETCT for predicting the major pathologic response to the neoadjuvant PD-1 blockade in resectable non-small cell lung cancer. Eur J Nucl Med Mol Imag (2020) 47:1209-19. doi: 10.1007/s00259-020-04711-3

69. Brown EG, Wood L, Wood S. The medical dictionary for regulatory activities (MedDRA). Drug Saf (1999) 20:109-17. doi: 10.2165/00002018-19992002000002

70. Common Terminology Criteria for Adverse Events (CTCAE). Available at: https://ctep.cancer.gov/protocolDevelopment/electronic_applications/ctc. htm (Accessed August 10th, 2020).

71. Xu C, Chen Y, Du X, Liu J, Huang C, Chen L, et al. Comparative safety of immune checkpoint inhibitors in cancer: systematic review and network meta-analysis. BMJ (2018) k4226. doi: 10.1136/bmj.k4226

72. Li J, Tao R, Fan L, Song Y, Hu Y, Zhang W, et al. Sintilimab for relapsed/ refractory $(\mathrm{r} / \mathrm{r})$ extranodal NK/T cell lymphoma (ENKTL): Extended 
follow-up on the multicenter, single-arm phase II trail (ORIENT-4). J Clin Oncol (2020) 38:8050. doi: 10.1200/JCO.2020.38.15_suppl.8050

73. Antonia SJ, Borghaei H, Ramalingam SS, Horn L, De Castro Carpeño J, Pluzanski A, et al. Four-year survival with nivolumab in patients with previously treated advanced non-small-cell lung cancer: a pooled analysis. Lancet Oncol (2019) 20:1395-408. doi: 10.1016/S1470-2045(19)30407-3

74. Garon EB, Rizvi NA, Hui R, Leighl N, Balmanoukian AS, Eder JP, et al. Pembrolizumab for the treatment of non-small-cell lung cancer. N Engl J Med (2015) 372:2018-28. doi: 10.1056/NEJMoa1501824

75. Mok TSK, Wu Y, Kudaba I, Kowalski DM, Cho BC, Turna HZ, et al. Pembrolizumab versus chemotherapy for previously untreated, PD-L1expressing, locally advanced or metastatic non-small-cell lung cancer (KEYNOTE-042): a randomised, open-label, controlled, phase 3 trial. Lancet (2019) 393:1819-30. doi: 10.1016/S0140-6736(18)32409-7

76. Herbst RS, Baas P, Kim DW, Felip E, Perez-Gracia JL, Han JY, et al. Pembrolizumab versus docetaxel for previously treated, PD-L1-positive, advanced non-small-cell lung cancer (KEYNOTE-010): a randomised controlled trial. Lancet (2016) 387:1540-50. doi: 10.1016/S0140-6736(15)01281-7

77. Reck M, Rodríguez-Abreu D, Robinson AG, Hui R, Csőszi T, Fülöp A, et al. Pembrolizumab versus Chemotherapy for PD-L1-Positive Non-Small-Cell Lung Cancer. New Engl J Med (2016) 375:1823-33. doi: 10.1056/NEJMoa1606774

78. Xing Q, Zhang Z, Lin Q, Shen L, Wang P, Zhang S, et al. Myositis-myasthenia gravis overlap syndrome complicated with myasthenia crisis and myocarditis associated with anti-programmed cell death-1 (sintilimab) therapy for lung adenocarcinoma. Ann Transl Med (2020) 8:250. doi: 10.21037/atm.2020.01.79

79. Wen L, Zou X, Chen Y, Bai X, Liang T. Sintilimab-Induced Autoimmune Diabetes in a Patient With the Anti-tumor Effect of Partial Regression. Front Immunol (2020) 11:2076. doi: 10.3389/fimmu.2020.02076
80. Gao C, Xu J, Han C, Wang L, Zhou W, Yu Q. An esophageal cancer case of cytokine release syndrome with multiple-organ injury induced by an anti-PD-1 drug: a case report. Ann Palliat Med (2020) 9:2393-9. doi: 10.21037/apm-20-1310

81. Ni J, Zhang L, Zhang X. Marked elevation of creatine phosphokinase alone caused by sintilimab - beware of hypothyroid myopathy. Eur J Cancer (2020) 128:57-9. doi: 10.1016/j.ejca.2019.12.030

82. Kang K, Zheng K, Zhang Y. Paraneoplastic Encephalitis and Enteric Neuropathy Associated With Anti-Hu Antibody in a Patient Following Immune-checkpoint Inhibitor Therapy. J Immunother (1997) (2020) 43:165-8. doi: 10.1097/CJI.0000000000000314

83. Hu J, Li Y, Chen X, Luo C, Zuo X. Pulmonary fibrosis and cytokine release syndrome after hyperactivation with sintilimab. J Clin Pharm Ther (2020). doi: $10.1111 /$ jcpt.13217

84. Chao-jun X, Ke-fu Y, Hui-xin J, Yu-han DU, Wei-xi C, Yue Z, et al. [Mini health technology assessment of five programmed cell death protein 1 inhibitors](in Chinese). Chin J Hosp Pharm (2020) 40:1638-42,1662. doi: 10.13286/j.1001-5213.2020.15.08

Conflict of Interest: The authors declare that the research was conducted in the absence of any commercial or financial relationships that could be construed as a potential conflict of interest.

Copyright (c) 2020 Zhang, Mai, Jiang and Geng. This is an open-access article distributed under the terms of the Creative Commons Attribution License (CC BY). The use, distribution or reproduction in other forums is permitted, provided the original author(s) and the copyright owner(s) are credited and that the original publication in this journal is cited, in accordance with accepted academic practice. No use, distribution or reproduction is permitted which does not comply with these terms. 